\title{
Design and performance studies of an MCP based pinhole camera for laser-produced plasma diagnostics
}

\author{
Y B S R PRASAD ${ }^{1}$, V K SENECHA', H S VORA ${ }^{2}$, R K KHARDEKAR ${ }^{1}$ \\ and $\mathrm{H}$ C PANT' \\ ${ }^{1}$ Laser Plasma Group, R \& D Laboratory "D", and 2 C V L Group, R \& D \\ Laboratory "C", Centre for Advanced Technology, Indore 452013, India \\ e-mail: yprasad@cat.ernet.in \\ MS received 17 December 1997; revised 19 February 1998
}

\begin{abstract}
An X-ray pinhole camera system has been developed and tested to obtain spatially resolved time-integrated X-ray images of laser-produced plasmas ( $\mathrm{Nd}$ : $Y A G$ laser of $75 \mathrm{~mJ}$ energy in 35 picoseconds) using a single stage micro channel plate (MCP) detector coupled to a CCD camera-frame grabber system instead of X-ray film. Thick slab targets of aluminium, copper and gold were used to produce plasmas with different $X$-ray yields at laser intensities ranging from $8 \times 10^{11}$ to $8 \times 10^{12} \mathrm{~W} / \mathrm{cm}^{2}$. MCP gain was increased with the help of biasing voltages to record low intensity X-ray images, which have been analysed using an image processing software "PROMISE" developed in-house. The experimental results are presented.
\end{abstract}

Keywords. Laser-produced plasmas; pinhole camera; micro channel plate detectors; X-ray imaging; spatial resolution.

\section{Introduction}

Spatially resolved optical/X-ray images provide important clues to study the evolution of the physical processes taking place in laser-produced plasmas, plasma focus devices, $z$-pinch plasmas, and X-ray lasers and in particular for inertial confinement fusion (ICF) studies. X-ray pinhole cameras are some of the most widely used diagnostic tools for studying the spatial evolution of plasmas. By using filters of different materials, maps of different temperature regions can be obtained with the help of pinhole cameras. These images are also useful in studying the effect of external parameters such as magnetic fields on plasma expansion, velocity profiles, jet formations, enhancement of X-ray emission and plasma flow control etc. (Begimkulov 1992; Pisarczyk 1992; Guskov 1994; Shukla

Abbreviations: $\mathrm{MCP}$ - micro channel plate detector assembly; CCD - charged couple device to record visible images electronically and store the information in the form of a matrix of intensities at each pixel 
1995). Conventionally X-ray films were being used as a medium for recording images. Such detection systems have severe limitations of operating above a certain level of X-ray intensity dictated by the sensitivity of the X-ray film used. The X-ray emitting region corresponding to low temperature plasma is not recorded at all. As the pinhole size is reduced for obtaining better spatial resolution, the $\mathrm{X}$-ray energy reaching the image plane is also drastically reduced, demanding the usage of high sensitivity X-ray films. Another problem associated with X-ray films is that the results can be obtained off line only after processing the film. This requires that after each laser shot the plasma chamber has to be opened, thus breaking the vacuum, which is quite time consuming and inconvenient. In order to overcome these problems, micro channel plate detectors (MCP) are used in place of X-ray films in many laser plasma laboratories (Koenig 1988).

The MCP is an array of many single channel electron multipliers bundled together (Wiza 1979). The semiconducting single channels are parallel to each other and are generally kept at some angle $\left(0^{\circ}-8^{\circ}\right)$ with respect to the MCP faces. They are sensitive to charged particles, ions and electromagnetic radiation. It is observed that the surface work function of the MCP is very low, making it sensitive to electromagnetic radiation even below $2000 \AA$. MCP's that are substantially more sensitive than X-ray films in the wavelength region of $2-100 \AA$ have been reported in the literature (Hailey 1985). In order to increase the sensitivity of MCP's to X-rays, a material of high photoelectric yield is deposited on the front surface and the channel walls of the MCP. Cesium iodide, magnesium fluoride and gold are suitable materials for this purpose. When X-ray radiation falls on the MCP, it generates electrons which are then accelerated towards the other end of the channel which is maintained at a higher potential. These elections generate secondary electrons upon hitting the channel walls, which are then directed towards a phosphor screen producing a visible image corresponding to the X-ray source. The main advantage of using the MCP's is their high sensitivity, the typical MCP gain varying between $10^{4}$ and $10^{6}$ (Fraser 1984). In order to increase the gain two micro channel plates are usually placed in tandem with a small gap between them. The channel axes of the two plates are biased at different angles (between $0^{\circ}$ and $8^{\circ}$ ) with respect to each other to prevent ion feedback. This is known as "Chevron configuration" or "double stage MCP". The MCP output images can then be coupled to a CCD camera/frame grabber system making the whole process on-line, with the advantage that the images can be captured, stored in the computer and processed subsequently for image analysis. For obtaining time resolved X-ray information from laser produced plasmas, framing cameras are used, in which independent strip electrodes coated on the MCP front surface are sequentially gated using fast ( $<1 \mathrm{~ns}$ ) high voltage pulsers (Koenig 1988).

In this article we report an indigenously developed MCP-based pinhole camera system for obtaining the spatially resolved, time integrated X-ray images of laser-produced plasmas on-line. We have attempted to study the spatial evolution of plasmas produced by irradiating slab targets of $\mathrm{Al}, \mathrm{Cu}$ and $\mathrm{Au}$ with a picosecond laser beam. The spatial extents and shapes of the plasma plumes under different experimental conditions are estimated. The choice of the targets was dictated by their different $\mathrm{X}$-ray yields. The experimental setup is described in $\S 2$. The results and discussion are presented in $\S 3$. 


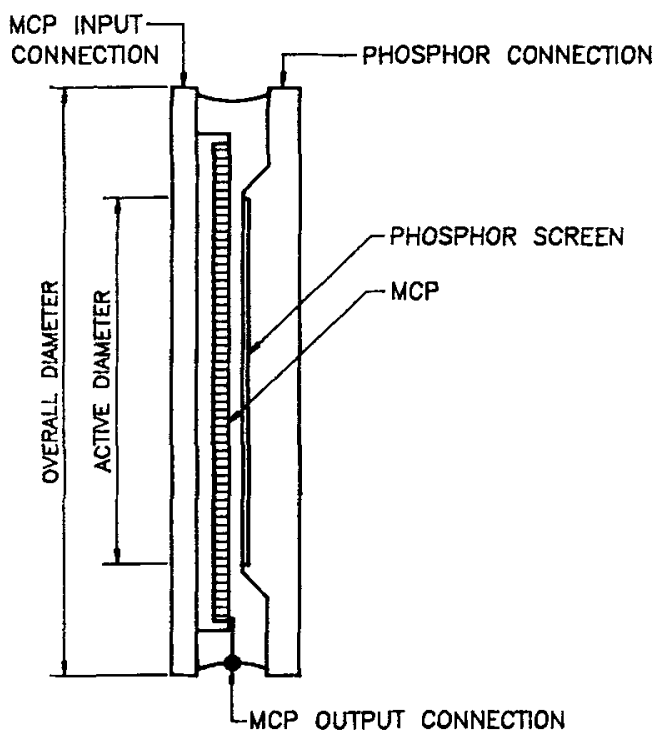

Figure 1. Schematic diagram of a single stage micro channel plate detector assembly.

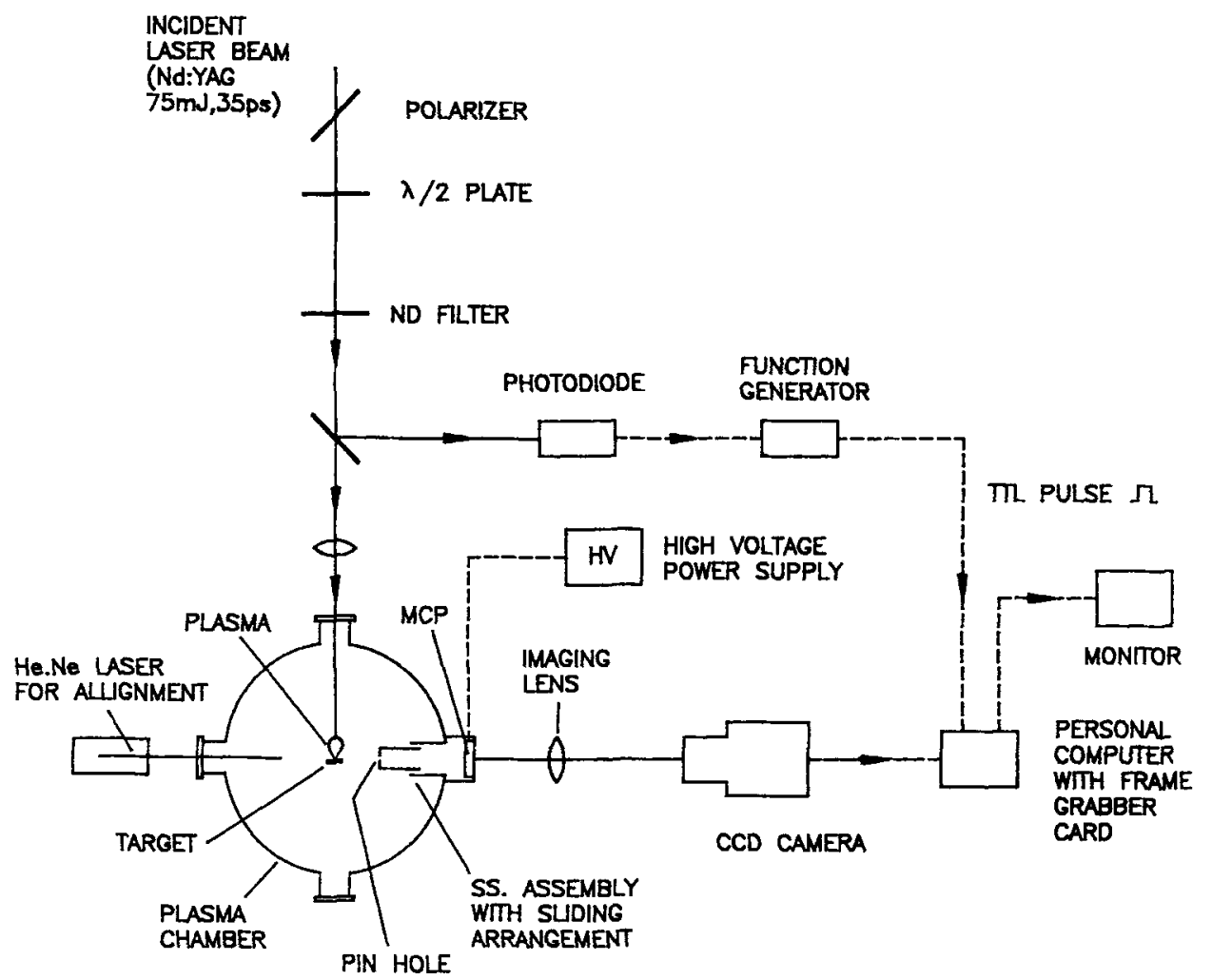

Figure 2. Schematic diagram showing experimental setup. 


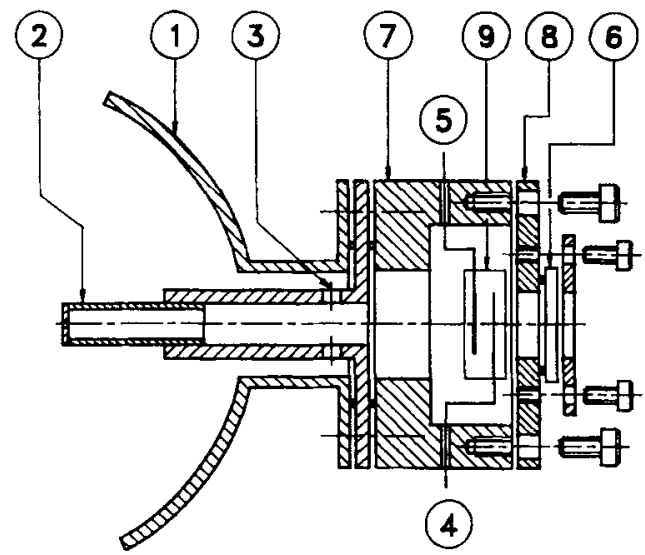

1 - PLASMA CHAMBER

2 - PIN HOLE AND SUDING TUBE ASSEMBLY

3 - EVACUATION HOLE

4 - PHOSPHOR

5 - MICRO CHANNEL PLATE

6 - BK7 WINDOW

7 - PERSPEX FLANGE FOR H.V CONNEGTIONS

8 - MCP HOLDING FLANGE (PERSPEX)

9 - MCP DETECTOR ASSEMELY

Figure 3. Schematic diagram showing the pinhole camera and frame grabber-CCD camera system.

\section{Experimental setup}

Mode locked laser pulses from a Nd:YAG picosecond laser system with $75 \mathrm{~mJ}$ energy in $35 \mathrm{pS}$ pulse duration are focused onto slab targets kept in a vacuum of $6 \times 10^{-6}$ torr with a lens of $30 \mathrm{~cm}$ focal length. It is essential to use vacuum of this order since high voltages are applied across the MCP surfaces. Poor vacuum will result in electrical discharges damaging the MCP surfaces. The typical focal spot size is about $\sim 150 \mu \mathrm{m}$ corresponding to laser intensity of $8 \times 10^{12} \mathrm{~W} / \mathrm{cm}^{2}$. The schematic diagram of an MCP detector is shown in figure 1. The pinhole camera and the frame grabber CCD camera system are shown in figures 2 and 3 . The pinhole assembly is provided with a sliding arrangement by which the distance from the source to the pinhole and the pinhole to the MCP can be varied without disturbing the alignment of the detector system. In the experimental setup we have used, the distance between the plasma source and pinhole $(u)=19 \mathrm{~mm}$ and the distance between the pinhole and MCP $(v)=180 \mathrm{~mm}$, giving a magnification factor of 9.5 . The $\mathrm{MCP}$ is attached to a port on the plasma chamber on a perspex flange having vacuum-sealed electrical connections. The pinhole $(30 \mu \mathrm{m})$ is covered with an aluminized poly carbonate foil (code name B-10) to block visible light. The overall thickness of the foil is $2 \mu \mathrm{m}$, both sides being coated with $\sim 700 \AA$ aluminium. It provides $1 / e$ transmission for an $\mathrm{X}$-ray energy of $\sim 1 \mathrm{keV}$.

Electrical connections to the MCP detector assembly are shown in figure 4. The maximum voltages prescribed between MCP-in and MCP-out and between MCP-out and the

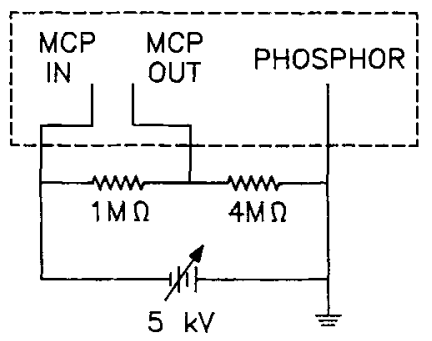

Figure 4. Electrical connection to the MCP assembly. 
phosphor screen are 1,000 volts/stage and 4000 volts respectively (Hamamatsu 1990). A $5 \mathrm{kV}$ dc power supply is used with a potential divider circuit to obtain requisite voltages for the MCP assembly. To reduce the dark current and to increase the lifetime of the MCP, the voltage applied between MCP-input and MCP-output can be gated.

The MCP output (visible image on the phosphor screen) is imaged on to a CCD camera and then grabbed by frame grabber system connected to a personal computer. Magnification factor of the CCD camera is calculated by imaging a measuring scale placed on the phosphor screen. It is found that each CCD pixel corresponds to $19 \mu \mathrm{m}$ on the phosphor screen and $2 \mu \mathrm{m}$ on the plasma source plane respectively. The whole system is synchronized with the laser pulse with the help of a photo diode detector system triggering a TTL pulse generator which in turn sends a "freeze" signal to the frame grabber system. The image can be viewed on a display monitor and stored in the computer for further analysis.

To study the performance of the MCP detector at different X-ray flux levels, the images of the laser-produced plasmas are recorded at different laser intensities. Laser intensity is varied with the help of neutral density filters. All other experimental conditions are kept constant throughout the experiment. The X-ray intensity $\left(I_{X}\right)$ varies with the laser intensity $\left(I_{L}\right)$ and the atomic number $(Z)$ of the target material, and can be expressed in the form of a scaling law

$$
\mathbf{I}_{\mathrm{X}} \propto\left(\mathbf{I}_{\mathrm{L}}\right)^{\mathbf{f}}
$$

where ' $f$ ', the scaling parameter is a function of the target atomic number $(Z)$. Typically $f=1.5-2.5$ (Eidmann 1991). When the X-ray fiux is very low the MCP gain can be increased by adjusting the biasing voltages for proper recording of the images. The gain of the MCP can be expressed as (Schagen 1974)

$$
G=\left(A V / 2 \alpha V_{0}^{1 / 2}\right)^{\beta} \text {, where } \beta=4 V_{0} \alpha^{2} / V
$$

(a)

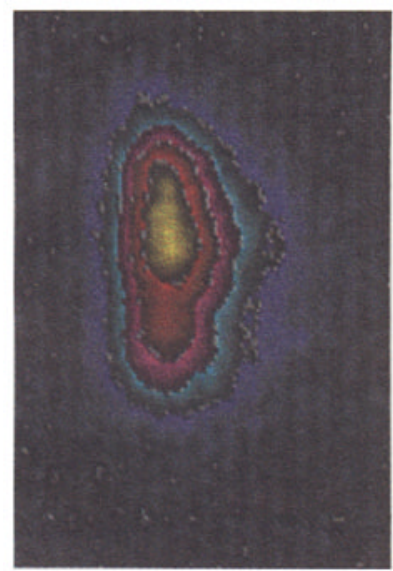

(b)

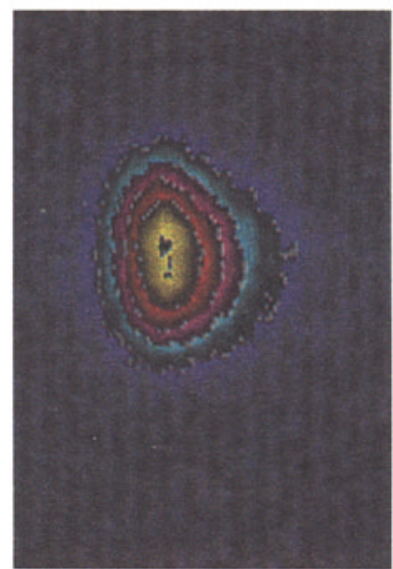

(c)

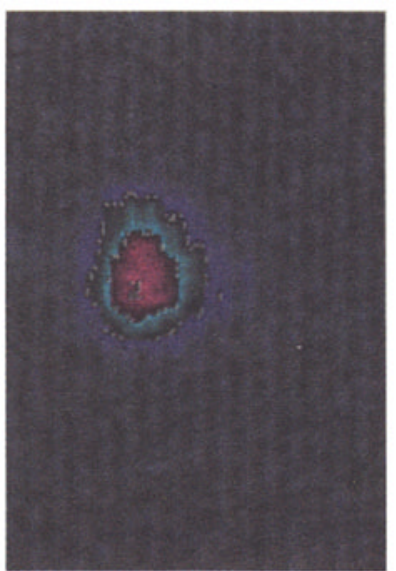

Figure 5. X-ray images of laser-produced plasma from aluminum target with B-10 filter at different laser intensities: (a) $8 \times 10^{12}$, (b) $6 \times 10^{12}$, (c) $8 \times 10^{11} \mathrm{~W} / \mathrm{cm}^{2}$. (Scale: figure a: height $300 \mu \mathrm{m}$ and width $145 \mu \mathrm{m}$.) 
Here $V$ is the total channel voltage $V_{0}$ is the initial energy of an emitted secondary electron $\sim 1 \mathrm{eV}, \alpha$ is the length to diameter ratio of channels $\sim 40-80$, and $A$ is the proportionality constant $\sim 0.2$.

\section{Results and discussion}

Plasma images produced under different experimental conditions such as variation in the laser energy, target material and $\mathrm{MCP} /$ phosphor voltages are shown in figures 5-7. As the laser pulse duration is very small, the plasma cools off rapidly and the expansion is limited which can be seen clearly in these figures (expansion length < plasma width). The plasma expansion length is about $50-100 \mu \mathrm{m}$ and the plasma width is about $200-300 \mu \mathrm{m}$. Even at very low laser intensities the $\mathrm{X}$-ray emissions can be properly recorded by suitably increasing the MCP/phosphor voltages. However, if the voltages are too high the phosphor images get saturated and voltages have to be adjusted accordingly.

In figures $5 \mathrm{a}-\mathrm{c}$ the $\mathrm{X}$-ray images from aluminum plasmas at laser intensities varying from $8 \times 10^{11}$ to $8 \times 10^{12} \mathrm{~W} / \mathrm{cm}^{2}$ are shown. Similarly figures 6 and 7 show $X$-ray images obtained from copper and gold plasmas respectively. Under very low irradiances or when the $\mathrm{X}$-ray emission in the wavelength interval we are considering is too low, it becomes difficult to record the images using conventional techniques on normal X-ray films. In the case of the MCP-based systems this problem can be overcome by operating the MCP at higher gain by increasing the MCP voltages. The results shown in figure 8 are recorded at a laser intensity of $8 \times 10^{11} \mathrm{~W} / \mathrm{cm}^{2}$ at different biasing voltages on the MCP detector assembly using two independent power supplies. It can be clearly seen from figure $8 \mathrm{a}$ that at biasing voltages $3000 / 750$ volts on the phosphor plate/MCP input the plasma image is very faint. So no useful information can be obtained from this'image. On the contrary at higher biasing voltages $3700 / 900$ and 3800/900 the images are quite intense and sharp

(a)

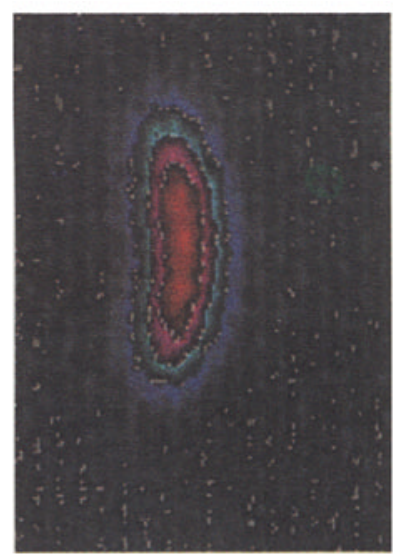

(b)

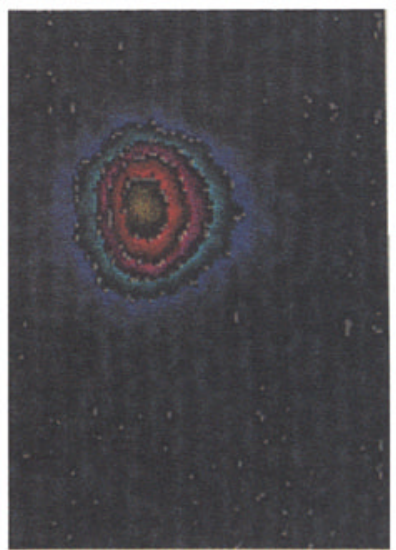

(c)

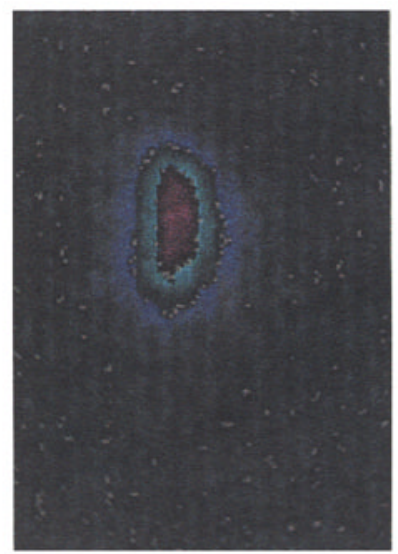

Figure 6. X-ray images of laser-produced plasma from copper target with B-10 filter at different laser intensities: (a) $8 \times 10^{12}$, (b) $6 \times 10^{12}$ (c) $8 \times 10^{11} \mathrm{~W} / \mathrm{cm}^{2}$. 
(a)

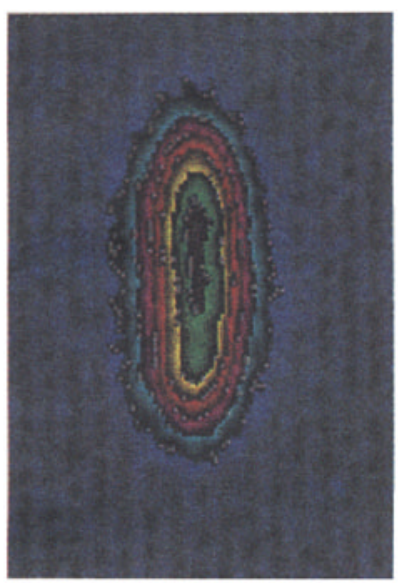

(b)

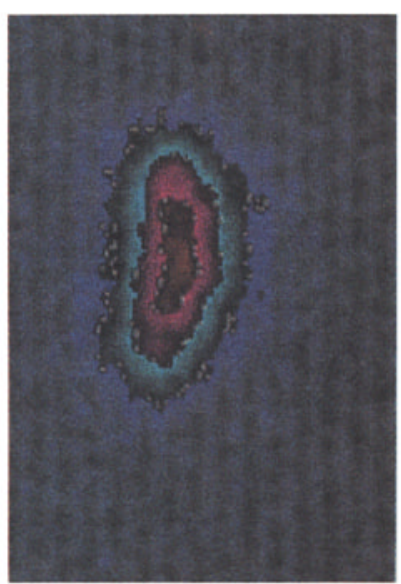

(c)

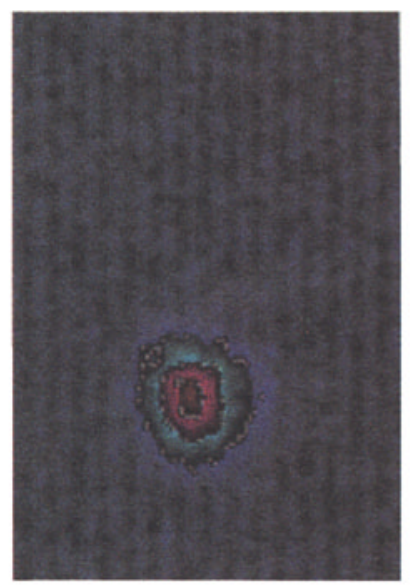

Figure 7. X-ray images of laser-produced plasma from gold target with B-10 filter at different laser intensities: (a) $8 \times 10^{12}$, (b) $6 \times 10^{12}$, (c) $8 \times 10^{11} \mathrm{~W} / \mathrm{cm}^{2}$.

as can be seen in figures $8 \mathrm{~b}$ and $\mathrm{c}$. The axial profiles of the images (figures 5a, 6a, 7a) obtained by using the "PROMISE" software (Vora 1996) are shown in figure 9. In this, the $X$-ray intensity is plotted in arbitrary units as a function of axial distance $Z$ from the target surface. In the case of monochrome CCD cameras the intensity of the light falling on the CCD is divided into 256 grey levels ( 8 bits) and is expressed in the range $0-255$.

The standard procedure for calibrating such devices is by imaging the spectrum from a known X-ray source such as a synchrotron radiation source. In the case of laser-produced plasmas these devices can be calibrated indirectly by using spectrographs (crystal or transmission grating type) with both MCP and X-ray films and comparing the results. But if the spectrum is not resolved, as is true in the present case, it is not possible to express the intensity distribution on the MCP image quantitatively since the X-ray spectrum emitted by the laser plasma is a strong function of the electron temperature. But for the purpose of comparing the spatial extents of X-ray emitting regions of plasma plume at different laser intensities for different target materials, arbitrary X-ray intensity scale is sufficient.

\section{Conclusions}

The MCP detector based X-ray pinhole camera system has been fabricated and successfully tested. X-ray images of the laser produced plasmas of aluminium copper and gold targets were obtained at different laser intensities. The detector has successfully recorded very

(a)

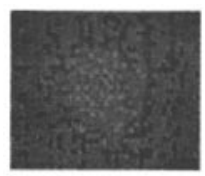

(b)

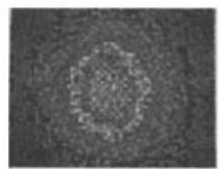

(c)

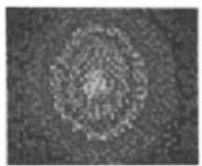

Figure 8. X-ray images from copper plasma at different MCP gains. 


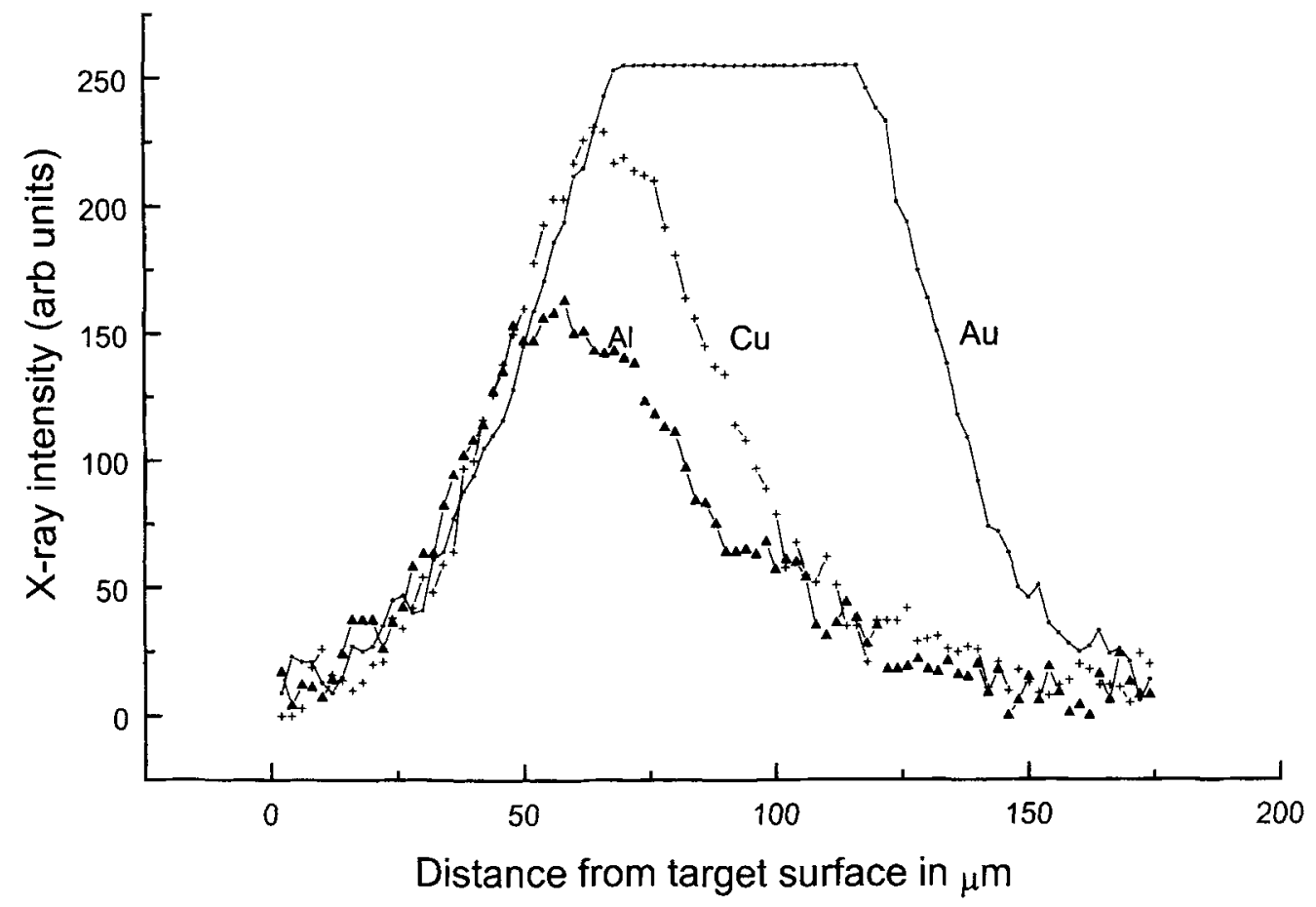

Figure 9. Axial profiles of X-ray images from three different targets at laser intensity $8 \times 10^{12} \mathrm{~W} / \mathrm{cm}^{2}$.

feeble X-ray images when operated at higher gains. This system can be used for studying the spatial evolution of laser-produced plasmas under different experimental conditions. Efforts are now being made to integrate a similar detection system with an X-ray spectrometer for spectral studies of plasma.

The authors would like to thank Dr V N Rai and Mayank Shukla for their help during the experiment. They would also like to thank Dr P A Naik for the helpful discussions held.

\section{Appendix A}

\section{A1 Spatial resolution of the pinhole camera}

The pinhole camera works on the principle that light travels in straight lines. This is known as the geometrical criterion. In this regime the spatial resolution $\mathrm{d} x$ of the pinhole camera system is expressed as

$$
\mathrm{d} x=a \times(1+(1 / M)),
$$

where $a$ is the pinhole aperture and $M$ is the magnification of the system defined as the ratio of image-pinhole distance $v$ and pinhole-object distance $u$. 


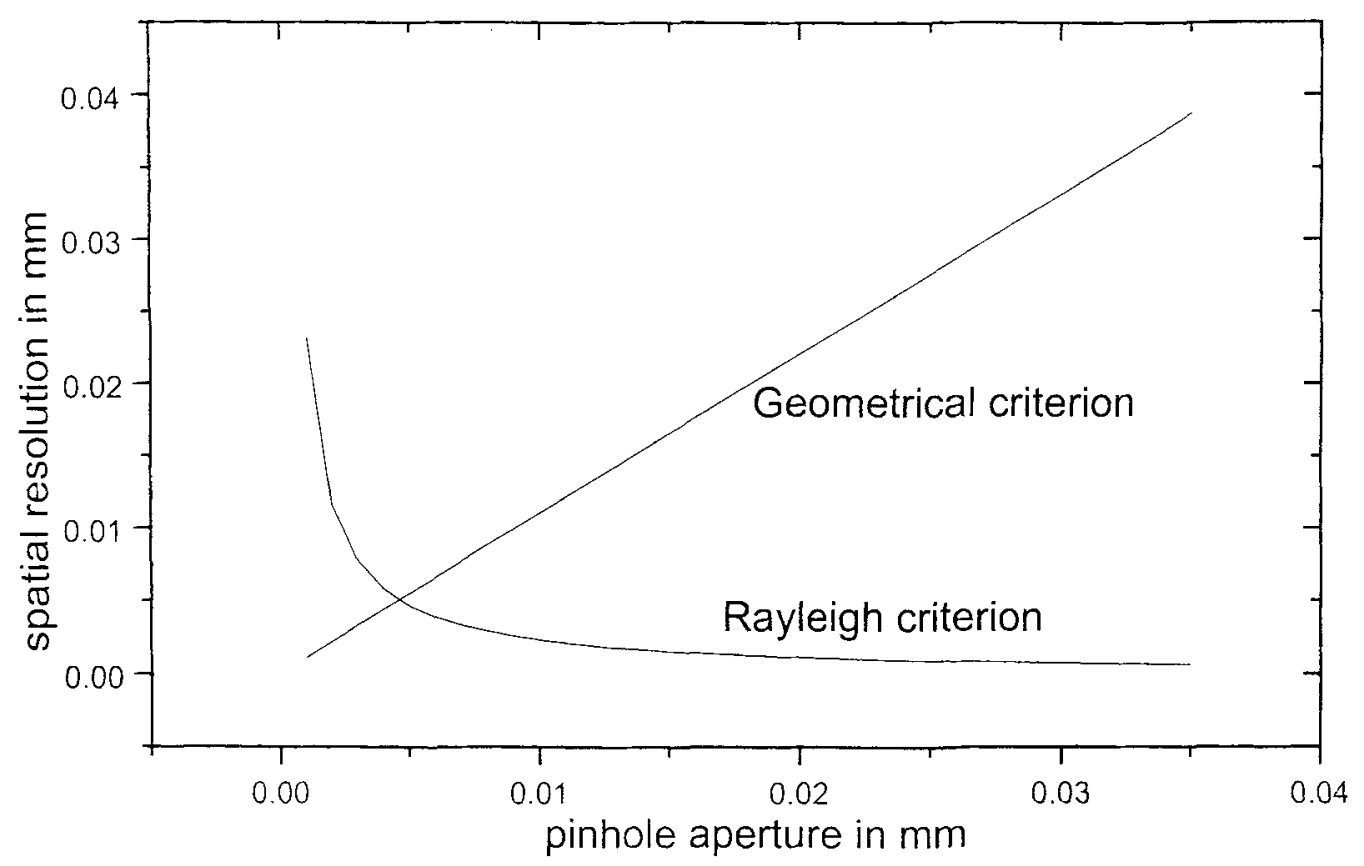

Figure A1. The spatial resolution of the pinhole camera as a function of pinhole aperture.

As the pinhole size is reduced, the image becomes more sharp until a situation arises when the pinhole becomes so small that diffraction effects becomes prominent. The resolution then deteriorates with decrease in the pinhole size. This is known as the "Rayleigh criterion", in which a point is cast as a diffraction pattern. When the central bright spot of one pattern coincides with the first minimum of the second, the points are at the limit of resolution. In this diffraction regime the spatial resolution $\mathrm{d} x$ can be expressed as

$$
\mathrm{d} x=(1.22 \times \lambda \times u) / a,
$$

where $\lambda$ is the wavelength of the radiation considered.

In the present case $u=19 \mathrm{~mm}, v=180 \mathrm{~mm}, a=30 \mu \mathrm{m}, M=9.5$, and the spatial resolution $\mathrm{d} x$ (geometrical regime) is $31.5 \mu \mathrm{m}$ and the spatial resolution (diffraction regime) is $0.78 \mu \mathrm{m}$ which means the spatial resolution of the system is $31.5 \mu \mathrm{m}$.

\section{A2 Optimum pinhole aperture}

Another point of interest is the optimum pinhole aperture $a$ that can be used in the present configuration for obtaining good spatial resolution, obtained by the following expression.

$$
a(\text { optimum })=[1.22 \times l \times u /(1+1 / M)]^{1 / 2} .
$$

In the present case $a$ (optimum) is $4.5 \mu \mathrm{m}$. Figure A1 shows the spatial resolution of the pinhole camera as a function of the pinhole aperture. But in the case of the systems we are considering here, i.e., MCP-based pinhole cameras, the channel diameter $(\sim 12 \mu \mathrm{m})$ and the CCD pixel size limit the overall spatial resolution of the system. 


\section{References}

Begimkulov U S 1992 Laser produced plasma expansion in a uniform magnetic field. Laser Part. Beams 10: 723-735

Eidmann K 1991 Conversion of laser light into soft X-rays with 3-ns and 30-ps laser pulses. Laser Part. Beams 9: 551-562

Fraser G W $1984 \mathrm{X}$ - and $\gamma$-ray imaging using microchannel plates. Nucl. Instrum. Methods 221: $115-130$

Guskov S Yu 1994 Magnetic control of the plasma flows in laser targets. Laser Part. Beams 12: 371-377

Hailey C J 1985 Microchannel plate intensified crystal spectrometer for use in soft X-ray spectroscopy. Rev. Sci. Instrum. 56: 1553-1556

Hamamatsu 1990 MCP assembly instruction manual

Koenig Z M 1988 Soft X-ray Bragg spectrograph with an intensified framing camera. Rev. Sci. Instrum. 59: 1813-1815

Pisarczyk T 1992 Formation of an elongated plasma column by magnetic confinement of a laserproduced plasma. Laser Part. Beams 10: 767-776

Schagen P 1974 Advances in image pickup and display (New York: Academic Press) vol. 1, p. 1

Shukla M 1995 X-ray enhancement in an external tranverse magnetic field confined laser produced plasma. Recent advances in plasma science and technology (PLASMA-95) (New Delhi: Allied Publishers) pp 187-194

Vora H S 1996 PROMISE - a software package for profile measurement. CAT Report No. CAT $/ 1996 / 10$

Wiza J L 1979 Micro channel plate detectors. (and another article) Nucl. Instrum. Methods 162: $587-601$ 